\title{
An La-related protein controls cell cycle arrest by nuclear retrograde transport of tRNAs during diapause formation in Artemia
}

\author{
Dian-Fu Chen ${ }^{1,2}$, Cheng Lin' ${ }^{1}$, Hong-Liang Wang ${ }^{1}$, Li Zhang ${ }^{1}$, Li Dai', Sheng-Nan Jia', Rong Zhou ${ }^{3}$, Ran Li ${ }^{4}$, \\ Jin-Shu Yang ${ }^{1}$, Fan Yang ${ }^{1}$, James S. Clegg ${ }^{5}$, Hiromichi Nagasawa ${ }^{1,6}$ and Wei-Jun Yang ${ }^{1 *}$
}

\begin{abstract}
Background: In eukaryotes, tRNA trafficking between the nucleus and cytoplasm is a complex process connected with cell cycle regulation. Such trafficking is therefore of fundamental importance in cell biology, and disruption of this process has grave consequences for cell viability and survival. To cope with harsh habitats, Artemia has evolved a special reproductive mode to release encysted embryos in which cell division can be maintained in a dormancy state for a long period.

Results: Using Artemia as a peculiar model of the cell cycle, an La-related protein from Artemia, named Ar-Larp, was found to bind to tRNA and accumulate in the nucleus, leading to cell cycle arrest and controlling the onset of diapause formation in Artemia. Furthermore, exogenous gene expression of Ar-Larp could induce cell cycle arrest in cancer cells and suppress tumor growth in a xenograft mouse model, similar to the results obtained in diapause embryos of Artemia. Our study of tRNA trafficking indicated that Ar-Larp controls cell cycle arrest by binding to tRNAs and influencing their retrograde movement from the cytoplasm to the nucleus, which is connected to pathways involved in cell cycle checkpoints.
\end{abstract}

Conclusions: These findings in Artemia offer new insights into the mechanism underlying cell cycle arrest regulation, as well as providing a potentially novel approach to study tRNA retrograde movement from the cytoplasm to the nucleus.

Keywords: Artemia, Cell cycle arrest, Diapause embryo, La-related protein, Nuclear retrograde of tRNA

\section{Background}

Regulation of the cell cycle involves processes crucial to survival, including the detection and repair of genetic damage and the prevention of uncontrolled cell division. This subject has attracted much attention and has enormous practical implications for combating cancer. The cell cycle has several well-controlled phases. Progression into each phase is tightly regulated by checkpoints and inhibitors $[1,2]$. The checkpoint allows the cell to repair DNA damage before entering mitosis, and studies of this

\footnotetext{
* Correspondence: w_jyang@zju.edu.cn

${ }^{1}$ College of Life Sciences, Zhejiang University, Hangzhou 310058, People's

Republic of China

Full list of author information is available at the end of the article
}

process may lead to chemotherapies with increased cytotoxicity. Two families, Cip/Kip and INK4a/ARF, prevent progression of the cell cycle. The Cip/Kip family includes p21, p27, and p57, which halt the cell cycle in G1 phase by binding to and inactivating cyclin-CDK complexes [3]. The INK4a/ARF family includes $\mathrm{p} 16^{\mathrm{INK} 4 \mathrm{a}}$, which binds to CDK4 and arrests the cell cycle in G1 phase, and p $14^{\text {Arf }}$ in humans, which prevents p53 degradation [1]. Each of these proteins acts through checkpoint signaling pathways that lead to cell cycle arrest [4]. In response to environmental stresses, such as starvation or treatment with thymidine or aphidicolin, cell cycle progress is halted in G1 phase by the control of multiple molecular pathways, rather than a single pathway [5]. 
In eukaryotes, tRNA trafficking between the nucleus and the cytoplasm is a complex process that often responds to environmental stress, thereby connecting transcription in the nucleus to translation in the cytoplasm [6]. This trafficking is of fundamental importance to cell biology, and disruption of this process has grave consequences for cell viability and survival. In response to genotoxic stress, regulation of tRNA trafficking constitutes an integral physiological adaptation to DNA damage at the cell cycle checkpoint. For example, in the yeast Saccharomyces cerevisiae, un-spliced tRNA rapidly accumulates in the nuclei, and cell cycle progression is delayed at the $\mathrm{G} 1$ to $\mathrm{S}$ phase transition $[7,8]$. In contrast to previous findings that showed that the transport of tRNA is unidirectional from the nucleus to the cytoplasm, recent studies have revealed that cytoplasmic tRNAs move retrogradely toward the nucleus and accumulate in response to nutrient deprivation [9-12]. Retrograde tRNA nuclear import might also serve to proofread tRNAs after splicing in order to separate improperly spliced tRNAs from the translation machinery [13]. The regulation of tRNA trafficking is dependent on the subcellular distribution of the elongation factor Tef1/2 (the yeast ortholog of eEF1 $\alpha$ ). The function of Tef1/2 is required for efficient tRNA nuclear export [14] and is dependent on Msn5 and Mtr10, as is the tRNA retrograde transport pathway [15].

La proteins are confined to the nucleus and the complete evolutionary in eukaryotic cells. Structural analysis of these proteins resulted in their classification into five families, namely the genuine La homologs and four Larp families: Larp1, 4, 6, and 7 [16, 17]. La proteins contain two RNA-binding motifs (RRMs) and an La motif, and have important functions in RNA metabolism. These proteins interact with an extensive variety of cellular RNAs, and exhibit activities to promote the metabolism of nascent pol III transcripts by binding to their common UUU-3'OH-containing ends and to modulate the translation of certain mRNAs involving an unknown binding mechanism [18].

The Artemia model studied here is found in severely hypersaline environments. Under certain conditions, mature females produce and release encysted gastrula embryos (also called cysts) that enter diapause, a state of obligate dormancy. Different environmental cues lead to uninterrupted (direct) embryonic development, resulting in the release of swimming nauplius larvae [19]. A feature of diapause embryos that is central to the present study is the complete absence of cell division and DNA synthesis during embryonic diapause [20, 21]. Diapause can be terminated by certain environmental conditions, leading to activated post-diapause embryos $[19,21]$. Remarkably, these activated encysted embryos develop without any DNA synthesis or cell division [22], and eventually hatch as nauplius larvae, at which point DNA synthesis and cell division resume [21, 23]. The Artemia model depicts adaptation as a complex response to critical life conditions, integrating and refining past and present experiences at all levels of organization [24].

To elucidate the molecular mechanism underlying cell cycle arrest and its link to the regulation of tRNA nucleocytoplasmic trafficking, Artemia diapause was used as a cell cycle arrest model. In this study, an RNAbinding and La-related protein, named Ar-Larp, was found to accumulate in the nucleus in response to cell cycle arrest, which resulted in the formation of Artemia diapause by binding to tRNAs. The mechanisms underlying the regulation of cell cycle arrest by Ar-Larp were elucidated in cancer cells using exogenous Ar-Larp gene transfection and expression. Cell cycle arrest induced by tRNA retrograde movement from the cytoplasm to the nucleus was then demonstrated in cancer cells. Our results indicated that tRNA trafficking regulates the mitogenesis and proliferation of cells through cell cycle checkpoints, a process that is mediated by multiple signaling pathways including histone $\mathrm{H} 3$ acetylated at lysine 56 (H3K56ac), extracellular signal-regulated kinase (ERK), and Akt. Ar-Larp is thus an upstream signal of tRNA trafficking that regulates cell cycle progression in response to environmental stresses.

\section{Results and discussion \\ Progress and characterization of cell cycle arrest during Artemia diapause formation}

As a survival strategy, Artemia possesses two independent reproductive pathways that allow adaptation to widely fluctuating environments. Under unfavorable conditions, mature females produce and release encysted embryos that enter diapause, a state of obligate dormancy (oviparous pathway; Fig. 1a). Alternatively, under favorable conditions, they release swimming nauplius larvae directly (ovoviviparous pathway; Fig. 1a). To determine the cell division state in each developmental stage, Western blotting was performed to analyze the expression of the mitosis markers CDK6, cyclin D3, phosphorylated $\mathrm{Rb}$ at Thr356, and phosphorylated histone $\mathrm{H} 3$ at Ser10, all of which were strongly inhibited in the diapause and post-diapause stages (Fig. 1b). The results suggested that the cell cycle ceased during the diapause and post-diapause stages compared with the prediapause and larval stages, in which cell division was widespread.

To distinguish the cell cycle phases of diapause and post-diapause embryos, which are characterized as nondividing cells, their DNA content was analyzed by flow cytometry. Analysis of the cell population distribution in diapause embryos revealed that more than $90 \%$ of cells were in G0/G1 phase, whereas in post-diapause 


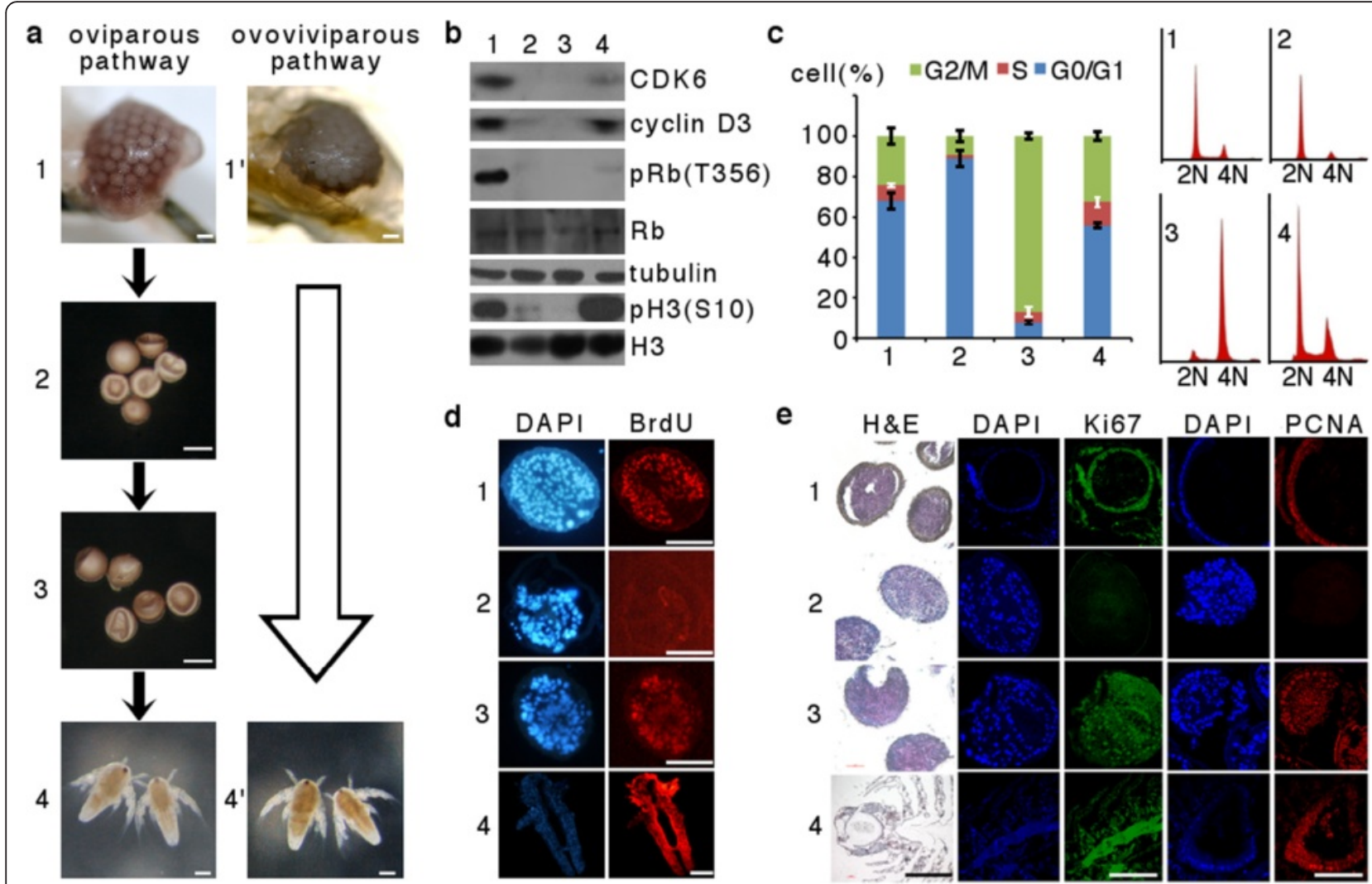

Fig. 1 Progression and characterization of cell cycle arrest during Artemia diapause embryo formation and termination. a Developmental stages of Artemia during diapause formation (oviparous pathway) and direct development (ovoviviparous pathway). 1, Pre-diapause (early embryos); 2, diapause; 3, post-diapause; 4, nauplii; 1', early embryos; 4', nauplii. Scale bar = $1 \mathrm{~mm}$. b Expression of the mitosis markers CDK6, cyclin D3, phosphorylated $\mathrm{Rb}$ (Thr356), and phosphorylated histone H3 (Ser10) at various stages of development. The lane numbers correspond to the developmental stages shown in (a). Histone $\mathrm{H3}(\mathrm{H} 3)$ and a-tubulin were used as the loading controls for the nucleus and cytoplasm, respectively. c Analysis of the cell cycle phase during various stages of development. Flow cytometry analysis was performed with a fixed cell suspension stained with PE at each stage. The right panel shows the DNA content of cells in each stage during diapause formation. d 5-bromo-2'-deoxyuridine (BrdU) incorporation assay and $\mathbf{e}$ immunofluorescence of the proliferation markers Ki67 and proliferating cell nuclear antigen at each stage of Artemia during diapause formation. Scale bar $=500 \mu \mathrm{m}$

embryos, more than $85 \%$ of cells were in G2/M phase, with very few cells in G0/G1 phase (Fig. 1c). These results were validated by the incorporation of 5-bromo-2'deoxyuridine (BrdU). The lack of any BrdU signal in diapause embryos revealed that the cell cycle had been arrested before $\mathrm{S}$ phase; in contrast, the signal could be detected in post-diapause embryos that had progressed to G2/M phase during activation (Fig. 1d). Furthermore, the proliferation markers Ki67 and proliferating cell nuclear antigen were not detected in cells of diapause embryos, but were detected in cells of pre-diapause and post-diapause embryos and nauplii (Fig. 1e).

\section{Characterization of a La-related protein from Artemia during diapause formation}

During Artemia diapause formation, a gene encoding an RNA-binding protein is expressed specifically in oocytes in the Artemia ovisac [25]. In the current study, the expression of this gene, named Ar-Larp, was characterized by real-time PCR. This showed that the gene was expressed strongly in diapause-destined Artemia, but at a very low level in the directly developing pathway. In the former, Ar-Larp gene expression levels increased during diapause formation, reaching a maximum level in the encysted gastrula. Ar-Larp expression then decreased in post-diapause gastrula and disappeared in hatched nauplii (Fig. 2a).

The Ar-Larp cDNA was cloned and sequenced to reveal a 1215 bp region with a single open reading frame encoding a 404 amino acid (aa) protein (Additional file 1: Figure S1). The molecular mass and isoelectric point of the Ar-Larp protein were calculated as $46.81 \mathrm{kDa}$ and 8.60 , respectively. According to the structure analysis, Ar-Larp contains an La motif (aa position 9-88) and two RNA recognition motifs, RRM1 (aa position 101-170) and RRM2 (aa position 282365), which are found in La and Larp proteins of many other species, ranging from trypanosomes to humans (Additional file 1: Figure S2). However, comparing the 


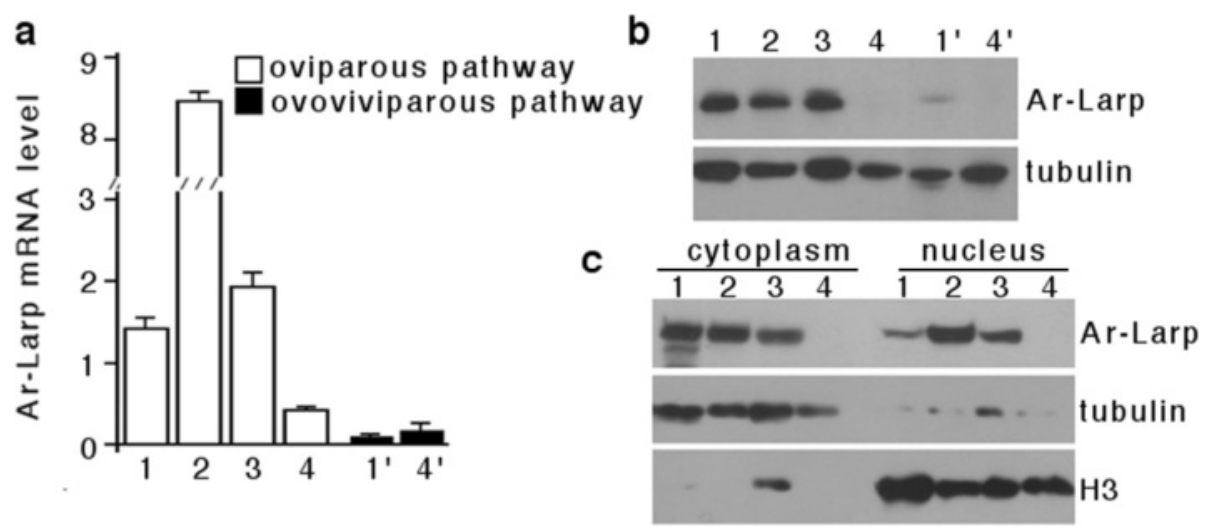

Fig. 2 Ar-Larp gene expression in each developmental stage of Artemia in the oviparous and ovoviviparous pathways. a Real-time quantitative PCR analysis of Ar-Larp expression during each developmental stage (indicated in Fig. 1a). Error bars represent the mean \pm SD of three independent experiments. b Western blot analysis of Ar-Larp during each developmental stage using a-tubulin as the loading control. c Subcellular location of Ar-Larp. Western blot analysis of Ar-Larp in the cytoplasm and nucleus during each developmental stage of the oviparous pathway. Histone H3 (H3) and $a$-tubulin served as the loading controls for the nuclear and cytoplasmic samples, respectively

sequence with other proteins in the DDBJ/EMBL/GenBank database revealed that Ar-Larp has very low sequence identity to other known proteins (less than $20 \%$ ).

Using polyclonal antibodies specific to Ar-Larp, a band of about $47 \mathrm{kDa}$ was identified in several stages of diapause-destined embryos, including oocytes and early, diapause, and post-diapause embryos, but not in hatched nauplii. In contrast, Ar-Larp was not detected in oocytes, embryos, or released nauplii of directly developing Artemia (Fig. 2b). In addition, Ar-Larp accumulated in the nuclei of diapause embryos, its level was decreased in the nuclei of post-diapause embryos, and it was undetectable in hatched nauplii (Fig. 2c). These results indicated that Ar-Larp is a diapause-specific protein. Furthermore, the nuclear accumulation and retention of Ar-Larp in diapause cysts of Artemia may have more important functions in cell cycle arrest during diapause embryo formation.

\section{Ar-Larp is required for diapause formation in Artemia and exhibits tRNA-binding activity}

RNA interference (RNAi) was used to knockdown Ar-Larp gene expression to elucidate the function of ArLarp during the regulation of cell cycle arrest at the onset of Artemia diapause. Just before ovarian development, a double-stranded RNA (dsRNA), designed from the ArLarp cDNA sequence, was injected into the body cavity of adult oviparous Artemia. One day after the formation of diapause embryos (about 2 weeks post-injection), Ar-Larp mRNA levels decreased in proportion to increasing doses of the dsRNA (Fig. 3a). After injection of $800 \mathrm{ng}$ of dsRNA, the Ar-Larp mRNA level was less than $15 \%$ of that in the control, and Ar-Larp protein expression remained undetectable by immunoblot analysis. In contrast to the control, neither the cell cycle nor embryogenesis were arrested in gene-knockdown embryonic cells. This prevented diapause formation and produced pseudodiapause, in which the embryos continued to develop and hatch into nauplii (Fig. 3b). In gene-knockdown embryos, the restriction of cyclin D3 and CDK6 expression and the block in phosphorylation of $\mathrm{Rb}$ (at Thr356 and Ser807/ 811) and histone H3 (at Ser10) were removed (Fig. 3c). These results strongly suggested that the knockdown prevented the formation of a complete diapause state, resulting in a pseudodiapause embryo in which the cell cycle was not arrested, and the embryos continued to develop and hatch into nauplii.

To understand Artemia diapause formation, previous studies have focused on molecules associated with extreme environmental tolerance. For example, the small heat shock/ $\alpha$-crystallin protein $\mathrm{p} 26$, which acts as a molecular chaperone, is expressed in diapause cysts and is transported into nuclei during periods of environmental stress [26, 27]. In addition, a cyst-specific protein, artemin, has high thermal stability and functions as an RNA chaperone in the cytoplasm $[28,29]$. In the current study, knockdown of the Ar-Larp gene prevented the formation of a complete diapause state and inhibited expression of p26 and artemin (Fig. 3c).

Based on our structural analysis, Ar-Larp contains a La motif and two RRM motifs, suggesting that the protein is capable of recognizing and binding to RNAs. Here, we examined the interaction between recombinant Ar-Larp (GST-Ar-Larp) and tRNAs using an electrophoretic mobility shift assay (EMSA). The results showed that GST-Ar-Larp could form a complex with tRNA in vitro, and the complex was identified as a super-shifted band when an anti-Ar-Larp antibody was 


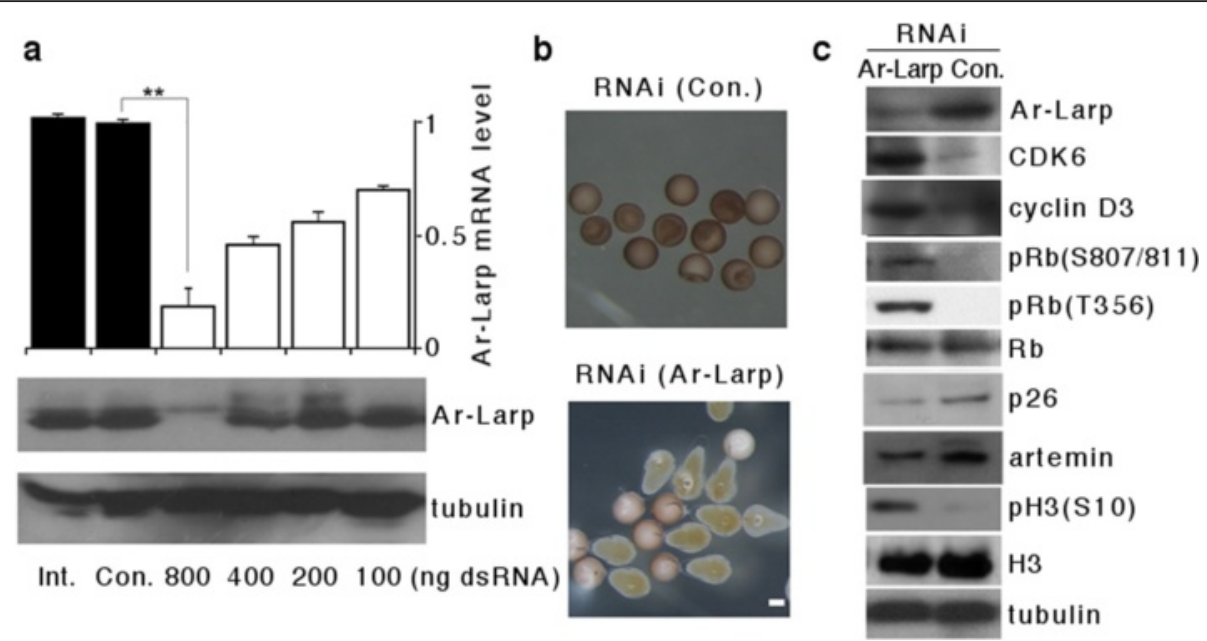

Fig. 3 RNA interference (RNAi) of Ar-Larp in Artemia. a The expression levels of Ar-Larp mRNA were determined by real-time quantitative PCR after RNAi treatments. Int, intact; Ctrl, control treatment with $800 \mathrm{ng}$ of GFP double stranded RNA (dsRNA). The proteins in diapause cysts were analyzed by Western blotting and tubulin was used as the loading control. b The phenotypes of RNAi-treated cysts injected with 800 ng of Ar-Larp dsRNA. Observation by the anatomical microscopy. Scale bar $=500 \mu \mathrm{m}$. Embryos treated with GFP dsRNA remained in diapause, whereas those treated with Ar-Larp dsRNA entered a pseudodiapause and developed into nauplii. c Western blot analysis of CDK6, cyclin D3, p26, Artemin, phosphorylated Rb (Thr356 and Ser807/811), and phosphorylated histone H3 (Ser10) after RNAi of Ar-Larp. Histone H3 (H3) and a-tubulin were used as the loading controls for the nucleus and cytoplasm, respectively

incubated together with Ar-Larp and tRNAs (Fig. 4a). To clarify the binding specificity of Ar-Larp to tRNA, several RNAs, including 5.8S rRNA, 5S rRNA, and mRNAs, were used as competitors of the binding. The result showed that none of these RNAs competed with the binding of Ar-Larp to tRNAs (Fig. 4b). Furthermore, the results of Northern blotting indicated that Ar-Larp could efficiently bind to both pre-tRNA and mature tRNA (Fig. 4c).

Both genetic and biochemical studies have revealed that a major role of $\mathrm{La}$ is to protect the 3' end of nascent small RNAs from exonuclease digestion [30, 31]. Although less is known about the roles played by the La protein binding to mature tRNAs, there is a report demonstrating LARP7mature tRNA interaction in vivo [32]. It should also be noted that Artemia oocytes are unusual cells, in that large quantities of proteins and RNAs are stored in preparation for early development. Thus, results obtained in Artemia oocytes may differ from other kinds of cells.

Next, co-immunoprecipitation (co-IP) was performed to investigate whether this interaction occurred in Artemia. Ar-Larp-tRNA complexes were identified in diapause embryos of Artemia and could be detected using several tRNA probes (Fig. 4d). However, the Ar-Larp-mRNA complex was not detected using probes specific for the mRNAs of tubulin, p26, and artemin, which are transcribed during diapause, indicating that Ar-Larp does not interact with mRNAs in Artemia (data not shown). Based on these results, we suggest that Ar-Larp controls cell cycle arrest in diapause by interacting with tRNAs in the nucleus.

\section{Ar-Larp localizes to the nucleus and induces cell cycle} arrest in cancer cells

To explore the function of Ar-Larp in the regulation of cell cycle arrest in systems other than Artemia, green fluorescent protein (GFP)-fused Ar-Larp was overexpressed in the human cancer cell lines HeLa (Fig. 5a) and MKN45 (Fig. 5b). Similar to the results obtained with the diapause embryos of Artemia, GFP-fused ArLarp localized to the nuclei in both of these cell lines (Fig. 5a,b). The results of the BrdU incorporation assay, the PKH26 label-retaining assay, and the expression of the proliferation marker Ki67 showed that, in contrast to control cells, cells overexpressing Ar-Larp had no mitotic activity (Fig. 5a,b). Flow cytometry analysis showed that most GFP-only transfected control cells progressed through $\mathrm{S}$ phase to G2/M phase, while GFP-Ar-Larptransfected cells did not progress to $G 2 / M$ phase and underwent cell cycle arrest (Fig. 5c). This result was also supported by a cell proliferation assay on soft agar, in which Ar-Larp clearly inhibited cell division and colony formation (Additional file 1: Figure S3). These results indicate that Ar-Larp induces cell cycle arrest in HeLa and MKN45 cells.

La-related proteins are conserved proteins with divergent structures and functions that interact with an extensive variety of cellular RNAs. Different La families may have evolved specialized activities in RNA metabolism [18]. Aberrant nuclear trafficking of La protein leads to the disordered processing of associated precursor tRNAs [31]. Although Ar-Larp has a low sequence 


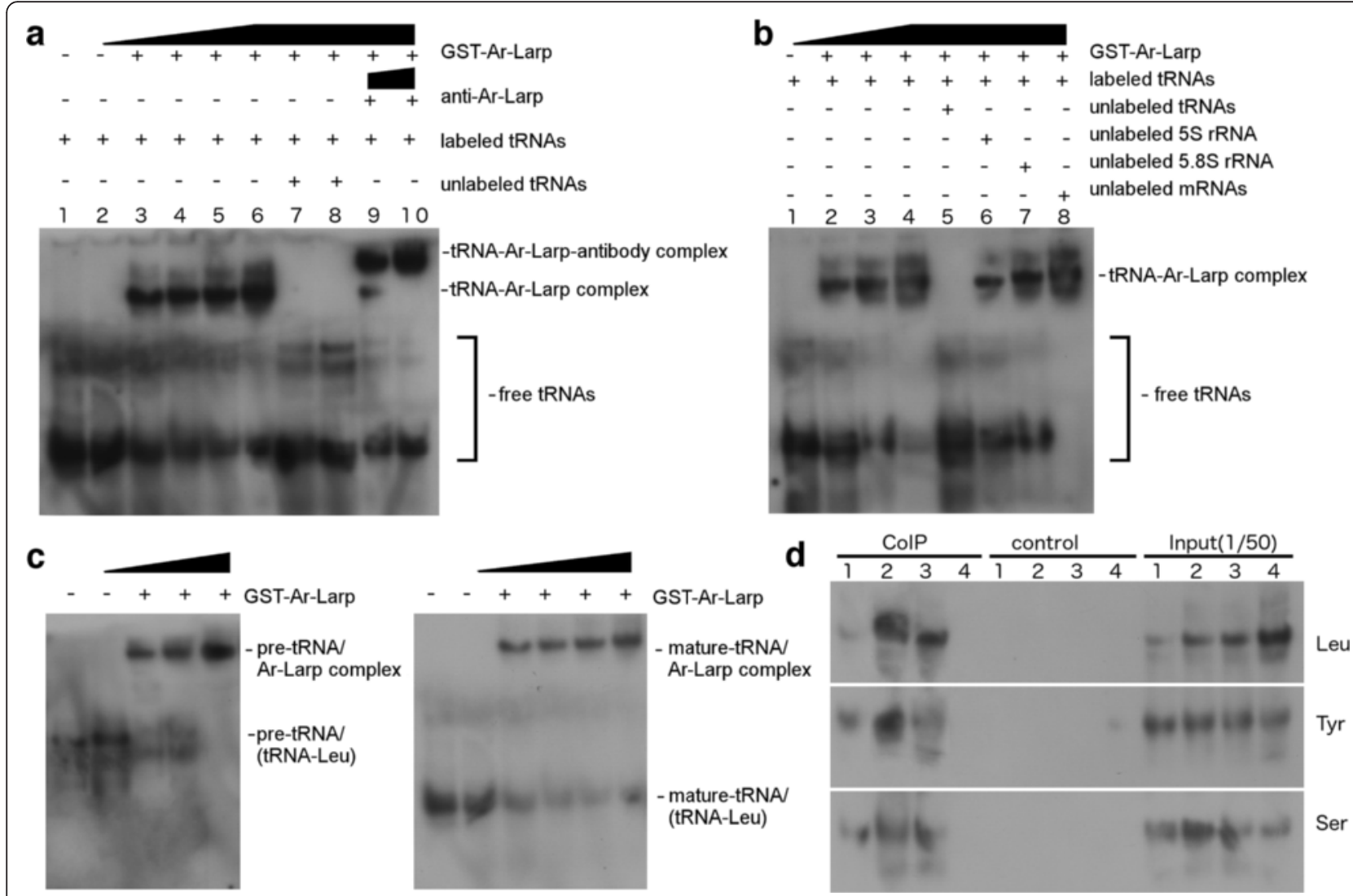

Fig. 4 Analysis of the tRNA-binding activity of Ar-Larp. a Electrophoretic mobility shift assay (EMSA) analysis of the affinity between Ar-Larp and tRNAs. All tRNAs (25 $\mathrm{\mu g}$ per lane) were labeled with digoxigenin (DIG) using the DIG Oligonucleotide 3'-End Labeling Kit. Lanes 2-5 contain increasing amounts $(0-1 \mu \mathrm{g})$ of GST-fused Ar-Larp. Ar-Larp-tRNA complexes were detected by Northern blotting ( 1 $\mu \mathrm{g}$ of GST was used as a control in lane 1). In lanes 7-8, excess tRNAs (250 and $500 \mu \mathrm{g}$, respectively) were added to the reaction system, which prohibited the binding of Ar-Larp to labeled tRNAs. In lanes 9-10, an anti-Ar-Larp antibody (0.5 and $2 \mu \mathrm{g}$, respectively) was added to the reaction system, and the super-shifted band of the Ar-Larp-anti-tRNA antibody complex was detected. b Competition EMSA analysis of the affinity between Ar-Larp and tRNAs and other RNAs. All tRNAs ( $25 \mu \mathrm{g}$ per lane) were labeled with DIG using the DIG Oligonucleotide 3'-End Labeling Kit. Lanes 1-4 contain increasing amounts (0-1 $\mu \mathrm{g})$ of GST-fused Ar-Larp. Ar-Larp-tRNA complexes were detected by Northern blotting. In lanes 5-8, excess RNAs (tRNA, $5.8 \mathrm{~s} r R N A, 5 \mathrm{~s} r R N A$, and mRNAs, $500 \mu \mathrm{g}$ each) were added to the reaction system as the competitors to prohibit the binding of Ar-Larp to labeled tRNAs. c EMSA analysis of the affinity between Ar-Larp and pre-tRNA and mature tRNA. Unlabeled tRNAs (25 $\mu \mathrm{g}$ per lane) were incubated with GST-Ar-Larp (0-1 $\mu \mathrm{g})$. Ar-Larp-tRNA complexes were detected using probes specific to pre-tRNA (tRNA ${ }^{\text {Leu }}$, c-left panel) and mature tRNA (tRNA ${ }^{\text {Leu }}$, c-right panel) (1 $\mathrm{\mu g}$ of GST was used as a control in lane 1). $\mathbf{d}$ Detection of Ar-Larp-tRNA complexes by co-IP using an anti-Ar-Larp antibody and probes specific to tRNAs. Northern blot analysis of tRNAs from the pellets (co-immunoprecipitation) or total extract fractions (Input) of Artemia cells at each developmental stage (indicated in Fig. 1a, isotype antibody was used as a control)

identity to other known Larps, we found that it contains an La motif and two RRMs that are conserved in the Larp family (Additional file 1: Figure S4). To identify the Ar-Larp motifs that are important for its nuclear localization, its tRNA-binding, and its ability to regulate cell cycle arrest, six Ar-Larp mutants with various motif deletions were constructed and purified. The results of the tRNA-binding activity of each mutant indicated that both RRM1 and RRM2 participate in the tRNA-specific binding activity of Ar-Larp (Additional file 1: Figure S5). The La motif of Ar-Larp did not show tRNA-binding activity, although it had typical secondary and tertiary structures (Additional file 1: Figure S4). However, when this motif was aligned with other known La proteins,
Phe residues at 23,33 , and 55 positions conserved in $\beta$-sheet 1 and 2 of the La motif in the proteins were changed to Leu, Val, and Cys residues in Ar-Larp. These conserved Phe residues are considered to be important for RNA recognition and tRNA-binding of the La motif in these proteins. Furthermore, the expression of the ArLarp mutants in HeLa cells suggested that the linker between RRM1 and RRM2 is associated with the retention of Ar-Larp in the nuclei of these cells (Additional file 1: Figure S6). Based on these results, six additional Ar-Larp mutants with various motif deletions were constructed and overexpressed in HeLa cells. BrdU incorporation was analyzed to detect the proliferation status. Mutants that had tRNA-binding activity without being retained in 


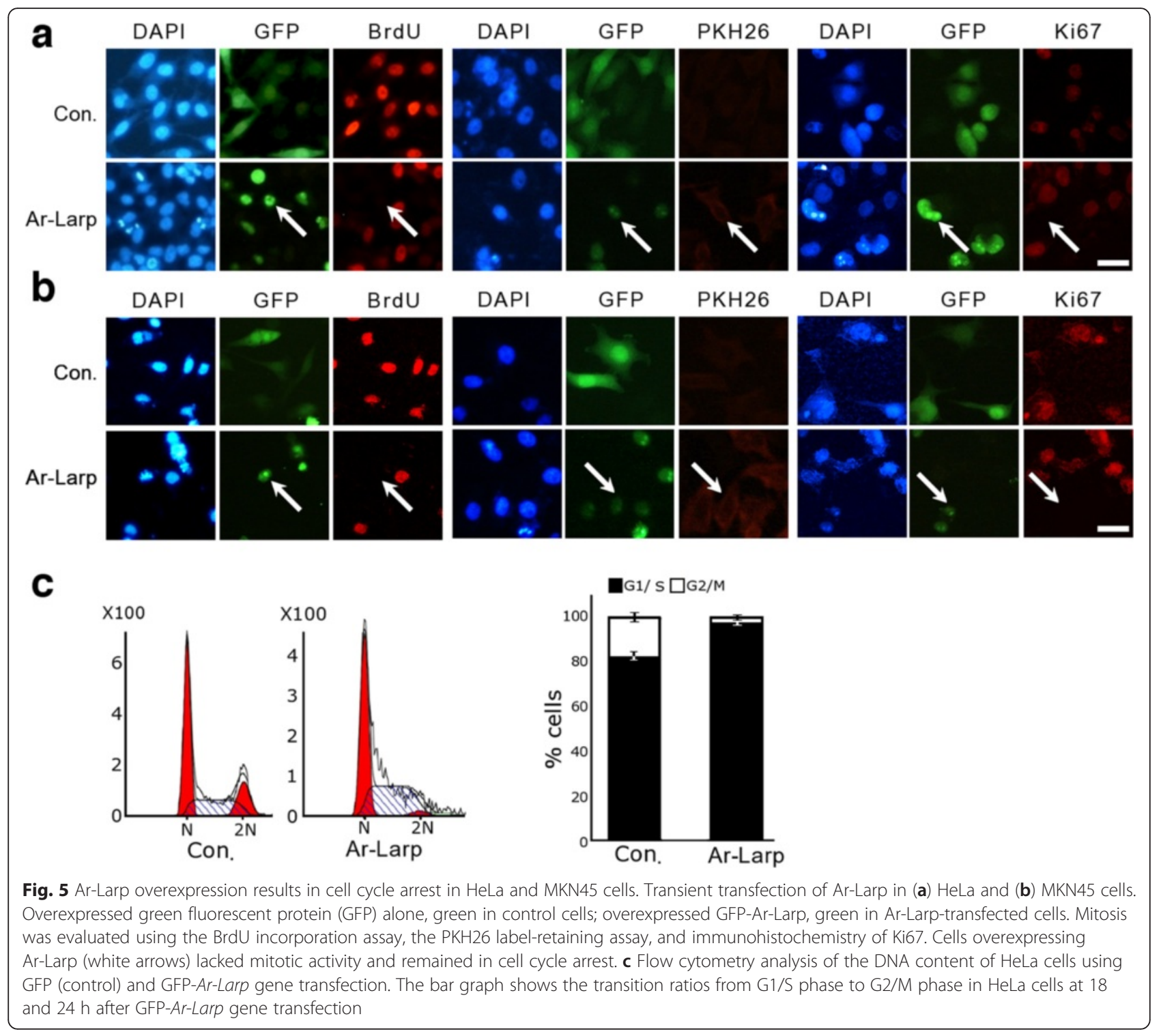

nuclei (Additional file 1: Figure S7a,b) or were retained in nuclei without tRNA-binding activity (Additional file 1: Figure S7e) could not induce cell quiescence. Only a combination of the nuclear retention (linker) and tRNAbinding (RRM1 and/or RRM2) regions could induce cell quiescence (Additional file 1: Figure S7c,d,f).

To investigate whether Ar-Larp could induce cell quiescence in a mouse tumor model, an Ar-Larp adenovirus (Ad-Ar-Larp) was constructed and injected into HeLa and HT1080 human tumor xenograft model mice. Western blotting revealed the presence of Ar-Larp in tumor specimens, indicating that the protein was successfully expressed from the adenovirus construct in vivo (Fig. 6a insertion). Ar-Larp suppressed the growth of both HeLa and HT1080 tumors, representing $56 \%$ and $55 \%$, respectively, of tumor formation in control mice (Fig. 6a). Importantly, BrdU labeling and detection revealed that all
Ar-Larp-expressing cells in both HeLa and HT1080 tumors showed no mitotic activity compared with their controls (Fig. 6b). Further analysis indicated that expression of cyclin D3, CDK4/6, and phosphorylated Rb was strongly inhibited in HeLa tumors expressing Ar-Larp in contrast to the controls (Fig. 6c). Based on these results, we concluded that Ar-Larp is capable of inducing cell cycle arrest in tumors as well as in Artemia embryos.

Ar-Larp controls cell cycle arrest by retrograde transport of tRNAs to the nucleus and its signaling pathways

To address the function of Ar-Larp in cell cycle regulation, GFP-fused Ar-Larp was transfected into HeLa cells and tRNA trafficking was analyzed. Fluorescence in situ hybridization (FISH) using tRNA-specific probes was used to examine the distribution of tRNAs. The results indicated that tRNAs accumulated significantly in the 


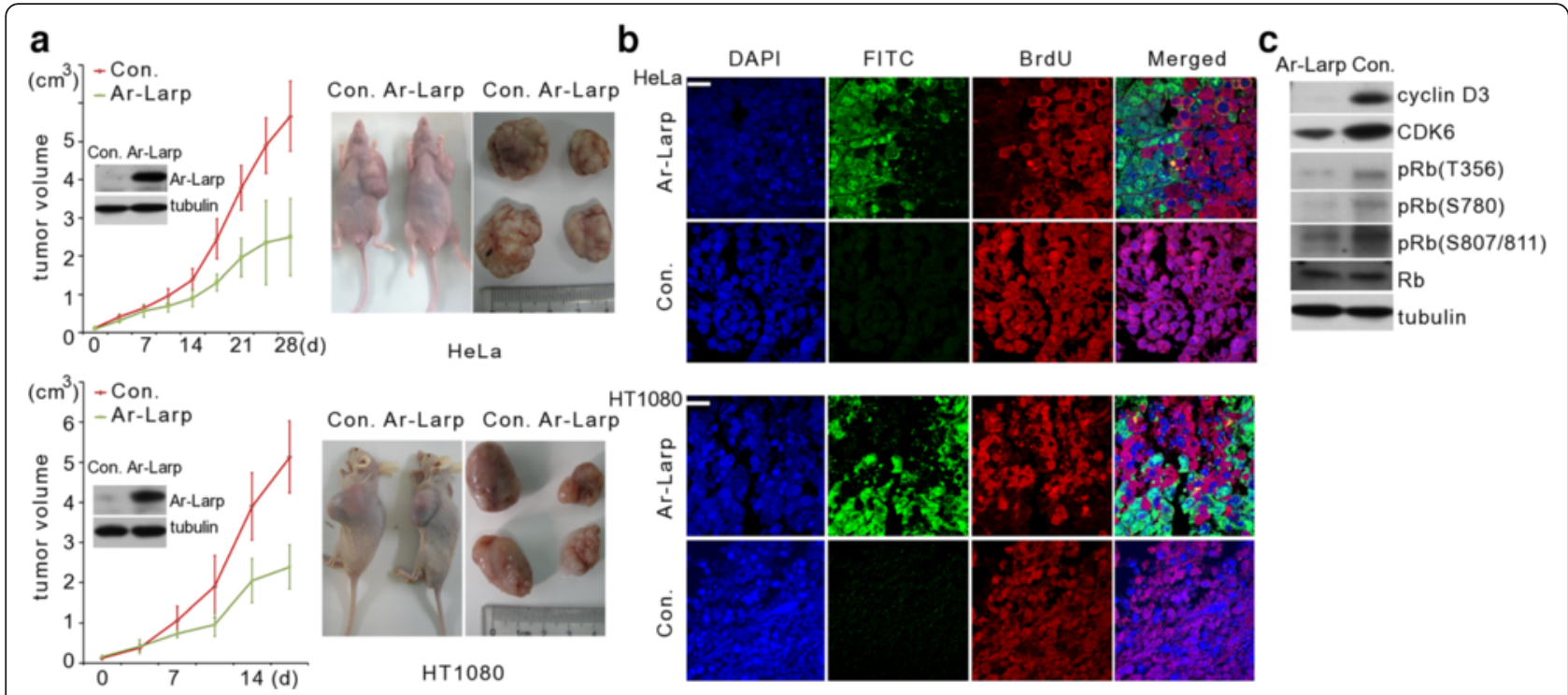

Fig. 6 Ar-Larp inhibits mitosis of HeLa and HT1080 cells in mouse xenograft tumor models. a Female BALB/c nude mice were intratumorally treated with Ad-Ar-Larp or Ad-control after the formation of HeLa or HT1080 tumors. Tumor volumes and images are shown. The error bars represent the mean \pm SD for five animals. Ar-Larp expression in the tumors was detected by Western blotting (insert). b Mitotic activity of cells in HeLa and HT1080 tumors examined using the BrdU incorporation assay after treatment with Ad-Ar-Larp or Ad-control. Blue, nuclei counterstained with DAPI. Green, Ar-Larp detected with a FITC-conjugated anti-Ar-Larp antibody. Red, incorporated BrdU detected with a rhodamine-conjugated anti-digoxigenin antibody. There is no mitotic activity in the green cells in the merged fields. Scale bar $=50 \mu \mathrm{m}$. $\mathbf{c}$ Expression of Ar-Larp and mitosis markers including CDK6, cyclin D3, and phosphorylated Rb (Thr356, Ser780, and Ser807/811). Tubulin was used as a loading control

nuclei of Ar-Larp-transfected HeLa cells compared to the controls (Fig. 7a). In addition, a co-IP assay was performed using an anti-Ar-Larp antibody to identify the tRNAs that accumulated in the nucleus. Immunoprecipitates were detected in cells transfected with the GFPfused Ar-Larp gene using several tRNA probes (Fig. 7b). The results suggested that Ar-Larp induces cell cycle arrest by regulating tRNA trafficking.

To address whether the accumulation of tRNAs in the nucleus was due to the suppression of trafficking to the cytoplasm or retrograde transport from the cytoplasm, actinomycin D (ActD, $5 \mu \mathrm{M}$ ) was used to block de novo tRNA synthesis. In complete medium, tRNA could not be detected in the nuclei of control cells treated with ActD; however, tRNA accumulation in the nuclei was observed in Ar-Larp-overexpressing cells treated with ActD (Fig. 7c). During the nuclear transport of Ar-Larp (from 18 to $36 \mathrm{~h}$ after expression), the accumulation and retention of tRNAs was increased. The results indicated that tRNAs accumulated in the nuclei of Ar-Larpoverexpressing cells by moving retrogradely from the cytoplasm through the nuclear transport of Ar-Larp.

Although the regulation of post-transcriptional gene expression by tRNA nuclear accumulation remains an unproven possibility, evidence indicates that retrograde tRNA nuclear accumulation occurs in response to environmental stress. Previous studies reported that tRNA moves retrogradely from the cytoplasm to the nucleus in rat hepatoma and S. cerevisiae cells in response to aa deprivation $[9,10,33,34]$. Thus, retrograde tRNA nuclear import may serve a regulatory role, separating tRNA from the translation machinery and resulting in the down-regulation of translation. However, the mechanism underlying tRNA nuclear import is unclear. In this report, our results indicate that Ar-Larp can function directly in retrograde tRNA nuclear import.

Based on the structural analysis, Ar-Larp from Artemia contains a La motif and two RRMs that are also found in La protein and LARP7. However, we found that Ar-Larp shows only $18 \%$ and $17 \%$ sequence identities to human La protein and LARP7, respectively (Additional file 1: Figure S8). Furthermore, human La protein and LARP7 did not show the activity of regulation in cell cycle arrest when they were overexpressed in HeLa cell line (Additional file 1: Figure S9). The previous report indicated that LARP7 inhibits breast cancer progression by binding to P-TEFb and 7SKsnRNA [35]. In this study, the complex of Ar-Larp binding with P-TEFb and 7SKsnRNA was not observed in Ar-Larp overexpressing HeLa cells by co-IP analysis and in vitro by EMSA analysis (Additional file 1: Figure S10). In addition, we did not find any homologue of Ar-Larp in HeLa cell by using anti-Ar-Larp antibody (Additional file 1: Figure S11). The results indicated that the structure and function of Ar-Larp is different to those of human La protein or LARP7 and the structure of molecule retrograded tRNA from cytoplasm 


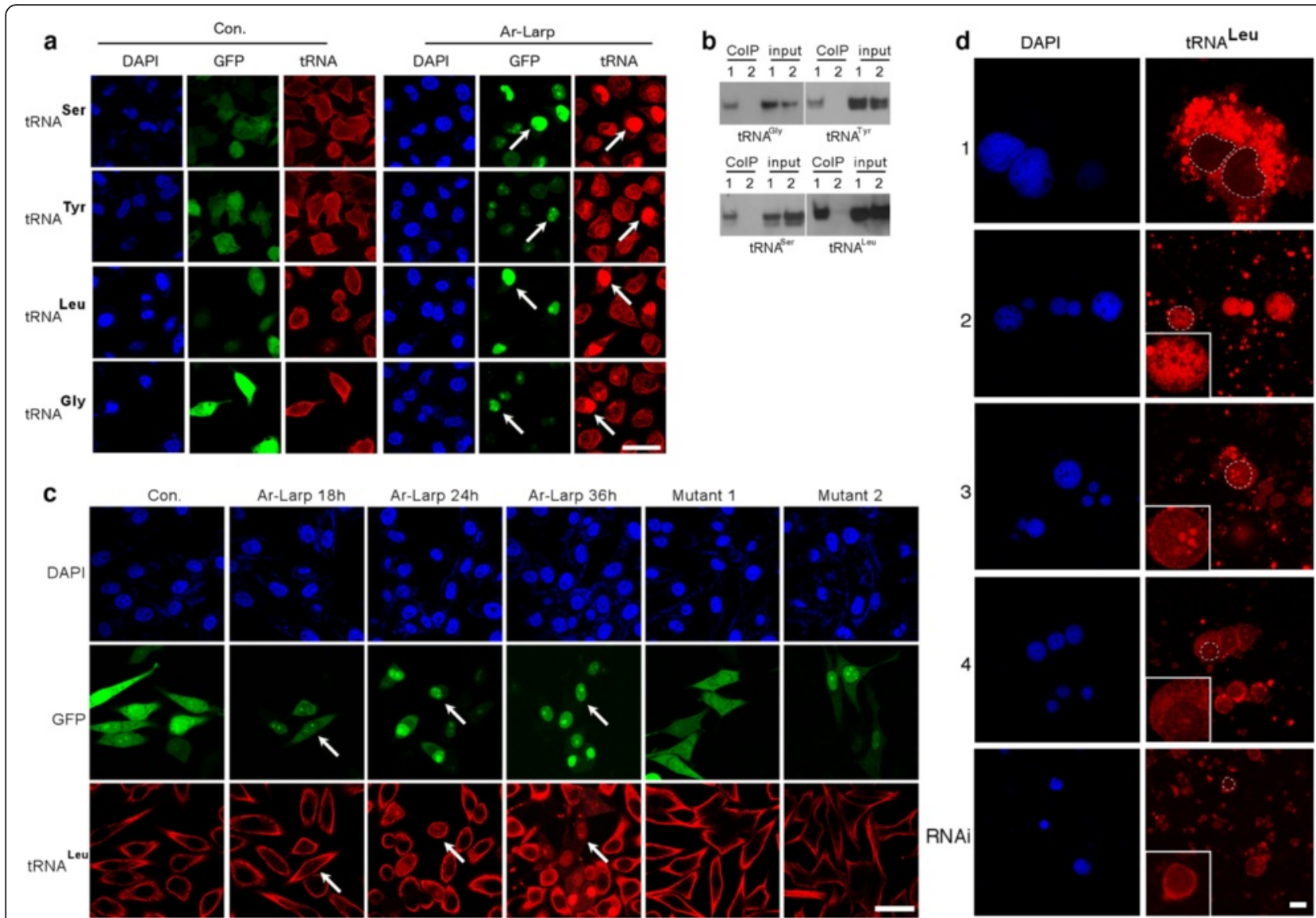

Fig. 7 Ar-Larp retrogradely transports tRNAs from the cytoplasm to the nucleus, resulting in cell cycle arrest. a The tRNA distribution in HeLa overexpressing GFP-Ar-Larp (white arrows indicate typical cells) or GFP alone. tRNAs were detected by FISH using oligonucleotide probes complementary to tRNA sequences. Green, GFP-fused Ar-Larp and GFP alone. Red, tRNAs detected with a rhodamine-conjugated anti-digoxigenin (anti-DIG) antibody. Blue, nuclei counterstained with DAPI. Scale bar $=50 \mu \mathrm{m}$. b Detection of Ar-Larp-tRNA complexes by co-immunoprecipitation using an anti-Ar-Larp antibody and probes specific to the tRNAs. Northern blot analysis of tRNAs from the pellets (co-IP) or total extract fractions (Input) of HeLa cells overexpressing GFP-Ar-Larp. Lane 1, HeLa cells overexpressing GFP-Ar-Larp; lane 2, control cells overexpressing GFP alone. c Analysis of the retrograde transport of tRNA from the cytoplasm to the nuclei in Ar-Larp-overexpressing HeLa cells. HeLa cells were treated with ActD $(5 \mu \mathrm{M})$ to block tRNA synthesis for $2 \mathrm{~h}$ after Ar-Larp was expressed for $8 \mathrm{~h}$. The nuclear and cytoplasmic distributions of tRNA in HeLa cells were then detected by FISH using oligonucleotide probes (tRNA ${ }^{\text {Leu }}$ ) complementary to the tRNA sequences at 18, 24, and 36 h after Ar-Larp expression (white arrow indicates typical cells). Cells expressing GFP alone and two Ar-Larp mutants were used as controls. Mutant 1 lacked the domain between RRM1 and RRM2, and therefore was not retained in the nucleus. Mutant 2 could accumulate and be retained in the nucleus, but lacked the RNA-binding domain. Green, GFP-fused Ar-Larp and GFP alone. Red, tRNAs detected with a rhodamine-conjugated anti-DIG antibody. Blue, nuclei counterstained with DAPI. Scale bar $=50 \mu \mathrm{m}$. $\mathbf{d}$ The distribution of tRNAs in cells at each stage during diapause formation (the stages are indicated in Fig. 1a and in pseudodiapause cysts induced by Ar-Larp RNA interference. Dashed line circles indicate cells in each stage and pseudodiapause cysts. Scale bar $=50 \mu \mathrm{m}$

to nucleus leading to cell cycle arrest in human maybe different with that of Ar-Larp.

Furthermore, we compared the effect of Ar-Larp and protein synthesis inhibitors, such as puromycin, cycloheximide, and fusidic acid, which block protein synthesis in the elongation or translocation steps. Unlike cell cycle arrest induced by Ar-Larp, tRNA retrograde movement from the cytoplasm to the nucleus was not observed in MKN45 cells treated with these inhibitors (Additional file 1: Figure S12).

The tRNA distribution in cells of each stage during diapause formation and in pseudodiapause cysts induced by Ar-Larp RNAi in Artemia was identified by FISH analysis using a tRNA ${ }^{\text {Leu }}$ probe (Fig. 7d). The result showed that the distribution of tRNAs coincided with the expression and distribution of ArLarp. In diapause cyst cells, tRNAs accumulated in nuclei; however, in pseudo diapause cyst cells induced by Ar-Larp RNAi (as shown in Fig. 3b), the accumulation of tRNAs in nuclei disappeared simultaneously (Fig. 7d). Based on the results obtained in this study, we propose that Ar-Larp regulates cell quiescence through its involvement in the retrograde nuclear accumulation of cytoplasmic tRNAs. 
In our previous studies, we found that mitogenactivated protein kinase-ERK-ribosomal S6 kinase 1 is inhibited and H3K56ac accumulates in diapause embryos $[23,36]$. To clarify the signaling pathways linking tRNA trafficking to the cell cycle checkpoint, the pathways concerned were analyzed by Western blotting. Of the various processes and pathways involved, the level of H3K56ac remained high, while the phosphorylation of ERK1/2 and Akt was strongly inhibited. In addition, the activation of FoxO3a was also inhibited in HeLa cells after Ar-Larp gene transfection (Additional file 1: Figure S13). However, Ar-Larp did not appear to have any effect on the molecules of the other tested signaling pathways (Additional file 1: Figure S14).

To confirm that Ar-Larp was responsible for activating certain signaling pathways in Artemia, RNAi was performed as described above. After knockdown of the Ar-Larp gene, the level of H3K56ac was reduced and phosphorylation of ERK1/2 and Akt was increased (Additional file 1: Figure S15). Based on these findings, we propose the molecular mechanism underlying ArLarp-induced cell cycle arrest, as identified in this study. Ar-Larp retrogradely transports tRNAs to the nucleus, followed by the up-regulation of $\mathrm{H} 3 \mathrm{~K} 56 \mathrm{ac}$, and the down-regulation of ERK1/2 and Akt phosphorylation and inhibition of FoxO3a activation. Consequently, the cyclin D3/CDK6 complex is inactivated, while $\mathrm{Rb}$ is maintained in an active state to perform its function as a tumor suppressor by inhibiting cell cycle progression.

\section{Conclusions}

The regulation of the cell cycle involves processes that are crucial to survival, including the detection and repair of genetic damage and the prevention of uncontrolled cell division. Encysted gastrula in diapause released from Artemia provides an excellent model system for studying cell biology and the biochemical adaptation to extreme environments. In this study, we used Artemia as a peculiar model for cell cycle studies, and describe in detail how the Artemia RNA-binding protein Ar-Larp controls the onset of Artemia diapause, induces cell cycle arrest at G1/S phase in human cancer cells, and suppresses tumor growth in a xenograft mouse model. From Artemia, we have learned considerably about how cysts protect the embryos from extremely harsh environments, utilizing the capacity to maintain the embryos in a state of cell cycle arrest for long periods of time. Unlike protein inhibitors, Ar-Larp controls tRNA nucleocytoplasmic trafficking, transducing the signal to the cell cycle checkpoints, including H3K56ac, ERK, and Akt, which suppresses cell division. In this sense, Ar-Larp is an extraordinary protein and will be extremely useful in providing further insights into the regulation of cell division. Based on the unique structure and biological function of the Ar-Larp molecule, Ar-Larp and its related processes should help to provide novel approaches to non-specific gene therapies for cancer treatment.

\section{Methods}

Animals and ethics statement

Diapause-destined Artemia (Artemia parthenogenetica) were raised in $8 \%(\mathrm{w} / \mathrm{v})$ artificial seawater (Blue Starfish) at $25^{\circ} \mathrm{C}$ on a 4L:20D cycle, while the directly developing Artemia were reared in $4 \%(\mathrm{w} / \mathrm{v})$ artificial seawater on a 16L:8D cycle. Chlorella powder (Fuqing King Dnarmsa Spirulina Co., Ltd.) was supplied as brine shrimp food. $\mathrm{BALB} / \mathrm{c}$ nude mice aged 6-8 weeks (Institution of Animal Research, Shanghai, China) were used as xenograft recipients for cancer cells. Animal experiments were approved and performed in accordance with the institutional guidelines for animal care of the animal ethics committee of Zhejiang University.

\section{Cell culture and transfection}

HeLa, HT1080, and MKN45 cells (ATCC, VA, USA) were cultured at $37{ }^{\circ} \mathrm{C}$ in $5 \% \mathrm{CO}_{2}$ in DMEM, RPMI1640, and EMEM (Genom, China), respectively, supplemented with $10 \%$ fetal bovine serum (Gibco, MT, USA). Cell transfections were performed with Lipofectamine 2000 (Invitrogen, CA, USA) as recommended by the manufacturer.

\section{Western blotting and antibodies}

Ar-Larp protein with His-tag was expressed in Escherichia coli BL21 (DE3) and purified using Ni-NTA Agarose (Qiagen, Germany). The anti-Ar-Larp antibody was raised in rabbits (HuaAn, China). For nuclear and cytoplasmic fractions, the extracts were prepared using NPE Nuclear and CPE Cytoplasmic Extraction Reagents (Bioteke, China) following the manufacturer's instructions. Proteins were extracted from each tissue using protein loading buffer $(0.2 \mathrm{mM}$ dithiothreitol, $4 \%(\mathrm{w} / \mathrm{v})$ sodium dodecyl sulfate (SDS), $0.2 \%(\mathrm{w} / \mathrm{v})$ bromophenol blue, and $20 \%(\mathrm{v} / \mathrm{v})$ glycerol prepared in $0.1 \mathrm{mM}$ Tris- $\mathrm{HCl}, \mathrm{pH}$ 6.8). Fifty micrograms of protein of each sample were separated on $12.5 \%$ SDS-PAGE gels and transferred to PVDF membranes (Millipore, MA, USA). The membranes were incubated with antibodies overnight at $4{ }^{\circ} \mathrm{C}$ and detected using the BM Chemiluminescence Western Blotting Kit (Roche, Germany) following the manufacturer's instructions.

\section{Flow cytometry analysis}

Fluorescence-activated cell sorting was performed as previously described [23]. Data analysis was accomplished with Cell Quest ${ }^{\mathrm{Ts}}$ software, version 3.1 (BD Biosciences, NJ, USA). All data are presented as the mean \pm SD. All statistical analyses were performed using 
the two-tailed paired Student's $t$-test. The differences were considered statistically significant at $P<0.01$.

\section{BrdU incorporation assay}

In vivo BrdU labeling was performed according to the manufacturer's instructions (Sigma, MO, USA). Cells were incubated with $12 \mathrm{mM}$ BrdU (Sigma) for an additional $24 \mathrm{~h}$ after transfection. The incorporation of BrdU into the genome was detected with a mouse anti-BrdU primary antibody (Sigma) followed by a secondary goat anti-mouse antibody conjugated to rhodamine (HuaAn). Wide-field fluorescence microscopy was used to obtain images.

\section{Quantitative real-time PCR}

Total RNA was isolated using TRIzol reagent (Invitrogen). cDNA was synthesized from $2 \mu \mathrm{g}$ of total RNA using a SuperScript ${ }^{\mathrm{m} w} /$ First-Strand cDNA Synthesis kit (Invitrogen) with Oligo(dT) primers. cDNA was diluted 10-fold and combined with SYBR Green Supermix (TaKaRa, Japan) for quantitative PCR. The relative abundance of mRNA was normalized to that of $\alpha$-tubulin. The results are reported as the mean $\pm \mathrm{SD}$ of three independent experiments. Comparisons between groups for statistical significance were performed with a two-tailed paired Student's $t$-test.

\section{RNAi of the Ar-Larp gene}

Recombinant plasmids expressing Ar-Larp dsRNAs were constructed and the dsRNAs were induced, expressed, and purified as described previously [23]. Ar-Larp dsRNA (800 ng) was injected into the reproductive segment of Artemia using an UltraMicroPump II equipped with a Micro4 ${ }^{\text {ma }}$ MicroSyringe Pump (World Precision Instruments, FL, USA). Similarly, GFP dsRNA was injected as the negative control.

\section{EMSA}

For the EMSA, yeast tRNAs (Ambion, USA) were first labeled with the digoxigenin (DIG) Oligonucleotide 3'End Labeling Kit (Roche, USA) and then incubated with various concentrations of a GST-Ar-Larp fusion protein $(0-1 \mu \mathrm{g})$ or GST alone $(1 \mu \mathrm{g})$ in $20 \mu \mathrm{L}$ of RNA-binding buffer (Tris (pH7.5) $0.1 \mathrm{M}, \mathrm{KCl} 0.5 \mathrm{M}$, dithiothreitol $10 \mathrm{mM}$, with diisodecyl glutarate) at room temperature for $30 \mathrm{~min}$. The reaction mixtures were electrophoresed on $12.5 \%$ non-denaturing polyacrylamide gels in Tris/ Borate/EDTA buffer, and detected using the Northern Blotting Kit (Roche, Germany) following the manufacturer's instructions. For the super-shift assay, an anti-ArLarp antibody $(0.5-1 \mu \mathrm{g})$ was incubated simultaneously in the EMSA system. For competition EMSA experiments, several excess competitors (10-fold unlabeled tRNA, 5.8S rRNA, 5S RNA, and mRNAs) were added to the EMSA system.

\section{Co-IP}

Crude protein extracts were prepared in the presence of proteinase inhibitors (Sangon, China) and the RiboLock ${ }^{\text {tm }}$ Ribonuclease Inhibitor (MBI, CT, USA), and were incubated with an anti-Ar-Larp antibody (HuaAn) at $4{ }^{\circ} \mathrm{C}$ overnight, followed by the addition of Protein A Dynabeads (Invitrogen) for $3 \mathrm{~h}$ at $4{ }^{\circ} \mathrm{C}$. The beads were washed with extraction buffer twice, and tRNAs were purified using TRIzol reagent (Invitrogen). RNA was loaded onto $8 \%$ urea denaturing polyacrylamide gels prior to hybridization with tRNA oligonucleotide probes at $39^{\circ} \mathrm{C}$.

\section{Mouse xenograft model}

A recombinant adenovirus expressing Ar-Larp (Ad-ArLarp) was constructed and purified using the Adenovirus Dual Expression Kit (TaKaRa) following the manufacturer's instructions. Tumor-bearing BALB/c nude mice were injected with Ad-Ar-Larp or a control adenovirus (Ad-control) twice a week. Tumor volumes were determined by measuring tumors in two dimensions as described previously [37]. Four weeks later, the mice were injected with $\mathrm{BrdU}(50 \mathrm{mg} / \mathrm{kg})$ and ten hours later, the mice were sacrificed and the tumors were harvested and paraffin-embedded. BrdU incorporation was detected as described above.

\section{FISH}

tRNA FISH was performed as described previously [38]. To block de novo tRNA synthesis, cells were treated with $5 \mu \mathrm{M}$ ActD (Sigma) for $2 \mathrm{~h}$ and then cultured in complete medium. Cells were fixed and incubated with 3 ' end DIG-labeled tRNA oligonucleotide probes for hybridization at $39^{\circ} \mathrm{C}$. A rhodamine-conjugated anti-DIG Fab fragment (Roche, Germany) was used to determine the tRNA abundance. The tRNA oligonucleotide probes are listed in Additional file 2: Supplemental Materials and Methods.

\section{Availability of data and materials}

Supporting information and additional methods can be found in Additional files 1, 2, and 3.

\section{Additional files}

Additional file 1: Figure S1. Nucleotide sequence of Ar-Larp CDNA and its deduced amino acid sequences. Figure S2. Sequence alignment of the La (LAM), RRM1 and RRM2 motifs of Ar-Larp with the LARP7 protein family. Figure S3. Proliferation of HeLa cells overexpressing Ar-Larp. Figure S4. Structural predictions for the Ar-Larp. Figure S5. The affinity of Ar-Larp mutants for tRNA was analyzed by electrophoretic mobility shift assay (EMSA). Figure S6. Subcellular locations of six Ar-Larp mutants. Figure S7. Analysis of mitosis of HeLa cells overexpressing Ar-Larp mutants by BrdU-incorporation assay. Figure S8. Sequence alignment of Ar-Larp with human La protein and LARP7 protein. Figure S9. Analysis of mitosis of HeLa cells overexpressing human La protein and LARP7 by BrdU-incorporation assay. Figure S10. The affinity of Ar-Larp to 7SKsnRNA 
was analyzed by co-immunoprecipitation and EMSA. Figure S11. Proteins in HeLa cells were detected by anti-Ar-Larp antibody. Figure S12. The comparison of tRNA distribution of MKN45 after Ar-Larp overexpression and treated with general protein synthesis inhibitors. Figure S13. Analysis of signaling pathways in Ar-Larp-induced cell cycle arrest. Figure S14. Western blot analysis of cell cycle regulation related signaling pathways in HeLa cells 24, 36 and $48 \mathrm{~h}$ after GFP-fused Ar-Larp (GFP-Ar-Larp) or GFP only (Control) gene transfection. Figure S15. Western blot analysis of pAkt (T308), pERK1/2 (T202/ 204) and H3K56ac in diapause embryos of Artemia after Ar-Larp RNA interference treatment. (PDF $1459 \mathrm{~kb})$

Additional file 2: Supplementary methods. (DOC $65 \mathrm{~kb}$ )

Additional file 3: Table S1. Primes used in this study. Table S2. Sequences obtained in this study. Table S3. Oligonucleotides used in this study. (DOC $74 \mathrm{~kb}$ )

\section{Competing interests}

The authors declare that they have no competing interests.

\section{Authors' contributions}

DFC and WJY conceived the study and designed the research. DFC, CL, HLW, $L Z, L D, S N J, R Z$, and RL designed and performed the experiments. JSY, FY, $\mathrm{HN}$, and JSC contributed new reagents/analytic tools. DFC and JSY analyzed data. DFC, HN, JSC, and WJY prepared and revised the paper. All authors read and approved the final manuscript.

\section{Acknowledgements}

This work was funded by the National Natural Science Foundation of China (41476106 and 31470437) and Research Project of Zhejiang Provincial Education Department (Y201430816)

\section{Author details}

'College of Life Sciences, Zhejiang University, Hangzhou 310058, People's Republic of China. ${ }^{2}$ Key Laboratory of Conservation Biology for Endangered Wildlife of the Ministry of Education, Zhejiang University, Hangzhou 310058, People's Republic of China. ${ }^{3}$ Key Laboratory of Protein Chemistry and Developmental Biology of the State Education Ministry of China, College of Life Sciences, Hunan Normal University, Changsha 410018, People's Republic of China. ${ }^{4}$ Tianjin Key Laboratory of Animal and Plant Resistance, College of Life Sciences, Tianjin Normal University, Tianjin 300387, People's Republic of China. ${ }^{5}$ Section of Molecular and Cellular Biology and Bodega Marine Laboratory, University of California, Davis, Bodega Bay, California 94923, USA. ${ }^{6}$ Department of Biological Chemistry, The University of Tokyo, Yayoi, Bunkyo, Tokyo 113-8657, Japan.

Received: 29 December 2015 Accepted: 18 February 2016 Published online: 03 March 2016

\section{References}

1. Sherr CJ. The Pezcoller lecture: cancer cell cycles revisited. Cancer Res. 2000;60(14):3689-95.

2. Senderowicz AM, Sausville EA. Preclinical and clinical development of cyclin-dependent kinase modulators. J Natl Cancer Inst. 2000;92:376-87.

3. Malumbres M, Barbacid M. Mammalian cyclin-dependent kinases. Trends Biochem Sci. 2005:30(11):630-41.

4. Malumbres M, Barbacid M. Cell cycle, CDKs and cancer: a changing paradigm. Nat Rev Cancer. 2009;9(3):153-66.

5. Werner-Washburne M, Braun E, Johnston GC, Singer RA. Stationary phase in the yeast Saccharomyces cerevisiae. Microbiol Rev. 1993:57(2):383-401.

6. Hopper AK, Shaheen HH. A decade of surprises for tRNA nuclearcytoplasmic dynamics. Trends Cell Biol. 2008;18(3):98-104.

7. Hopper AK, Phizicky EM. tRNA transfers to the limelight. Genes Dev. 2003;17(2):162-80

8. Ghavidel A, Kislinger T, Pogoutse O, Sopko R, Jurisica I, Emili A. Impaired tRNA nuclear export links DNA damage and cell cycle checkpoint. Cell. 2007:131(5):915-26.

9. Shaheen $\mathrm{HH}$, Hopper AK. Retrograde movement of tRNAs from the cytoplasm to the nucleus in Saccharomyces cerevisiae. Proc Natl Acad Sci U S A 2005;102(32):11290-5

10. Shaheen HH, Horetsky RL, Kimball SR, Murthi A, Jefferson LS, Hopper AK Retrograde nuclear accumulation of cytoplasmic tRNA in rat hepatoma cells in response to amino acid deprivation. Proc Natl Acad Sci U S A. 2007:104(21):8845-50.

11. Whitney M, Hurto R, Shaheen H, Hopper A. Rapid and reversible nuclear accumulation of cytoplasmic tRNA in response to nutrient availability. Mo Biol Cell. 2007;18(7):2678-86

12. Huynh L, Thangavel M, Chen T, Cottrell R, Mitchell J, Praetorius-lbba M. Linking tRNA localization with activation of nutritional stress responses. Cell Cycle. 2010;9(15):3112-8.

13. Kramer E, Hopper A. Retrograde transfer RNA nuclear import provides a new level of tRNA quality control in Saccharomyces cerevisiae. Proc Natl Acad Sci U S A. 2013;110(52):21042-7.

14. McGuire A, Mangroo D. Cex1p is a novel cytoplasmic component of the Saccharomyces cerevisiae nuclear tRNA export machinery. EMBO J. 2007;26(2):288-300.

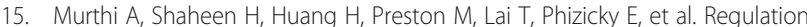
of tRNA Bidirectional Nuclear-Cytoplasmic Trafficking in Saccharomyces cerevisiae. Mol Biol Cell. 2010;21(4):639-49.

16. Wolin SL, Cedervall T. The La protein. Annu Rev Biochem. 2002;71:375-403.

17. Bousquet-Antonelli C, Deragon JM. A comprehensive analysis of the La-motif protein superfamily. RNA. 2009;15(5):750-64.

18. Bayfield MA, Yang R, Maraia RJ. Conserved and divergent features of the structure and function of La and La-related proteins (LARPS). Biochim Biophys Acta. 2010;1799(5-6):365-78.

19. MacRae TH. Diapause: diverse states of developmental and metabolic arrest. J Biol Res. 2005;3:3-14.

20. Nakanishi YH, Iwasaki T, Kato H. Cytological studies of Artemia salina. I. Embryonic development without cell multiplication after the blastula stage in encysted dry eggs. Annot Zool Japan. 1962;35:223-8.

21. Olson CS, Clegg JS. Cell division during the development of Artemia salina. Roux'sArch Dev Biol. 1978:184:1-13.

22. Clegg JS, Trotman CNA. Physiological and Biochemical Aspect of Artemia Ecology. In: Abatzopoulos THJ, Beardmore JA, Clegg JS, Sorgeloos P, editors. Artemia: Basic and Applied Biology. Berlin: Kluwer Academic Publishers; 2002. p. 129-70.

23. Dai JQ, Zhu XJ, Liu FQ, Xiang JH, Nagasawa H, Yang WJ. Involvement of p90 ribosomal $\mathrm{S} 6$ kinase in termination of cell cycle arrest during development of Artemia-encysted embryos. J Biol Chem. 2008;283(3):1705-12.

24. Gajardo GM, Beardmore JA. The brine shrimp Artemia: adapted to critical life conditions. Front Physiol. 2012;3:185.

25. Qiu Z, Tsoi S, MacRae T. Gene expression in diapause-destined embryos of the crustacean, Artemia franciscana. Mech Dev. 2007;124(11-12):856-67.

26. Clegg J, Jackson S, Liang P, MacRae T. Nuclear-Cytoplasmic translocations of protein p26 during aerobic-anoxic transitions in embryos of Artemiafranciscana. Exp Cell Res. 1995;219:1-7.

27. Liang P, MacRae T. The synthesis of a small heat shock/alpha-crystallin protein in Artemia and its relationship to stress tolerance during development. Dev Biol. 1999:207(2):445-56.

28. Warner A, Brunet R, MacRae T, Clegg J. Artemin is an RNA-binding protein with high thermal stability and potential RNA chaperone activity. Arch Biochem Biophys. 2004;424(2):189-200

29. Chen T, Villeneuve T, Garant K, Amons R, MacRae TH. Functional characterization of artemin, a ferritin homolog synthesized in Artemia embryos during encystment and diapause. FEBS J. 2007;274(4):1093-101.

30. Fan H, Goodier JL, Chamberlain JR, Engelke DR, Maraia RJ. 5' processing of tRNA precursors can be modulated by the human La antigen phosphoprotein. Mol Cell Biol. 1998;18(6):3201-11.

31. Xue D, Rubinson DA, Pannone BK, Yoo CJ, Wolin SL. U snRNP assembly in yeast involves the La protein. EMBO J. 2000;19(7):1650-60.

32. Hussain RH, Zawawi M, Bayfield MA. Conservation of RNA chaperone activity of the human La-related proteins 4, 6 and 7. Nucleic Acids Res. 2013;41(18):8715-25.

33. Intine RV, Dundr M, Mistelli T, Maraia RJ. Aberrant nuclear trafficking of La protein leads to disordered processing of associated precursor tRNAs. Mol Cell. 2002:9(5):1113-23.

34. Takano A, Endo T, Yoshihisa T. tRNA actively shuttles between the nucleus and cytosol in yeast. Science. 2005;309(5731):140-2.

35. Ji X, Lu H, Zhou Q, Luo K. LARP7 suppresses P-TEFb activity to inhibit breast cancer progression and metastasis. Elife. 2014:3:e02907.

36. Zhou R, Yang F, Chen DF, Sun YX, Yang JS, Yang WJ. Acetylation of chromatin-associated histone $\mathrm{H} 3$ lysine 56 inhibits the development of encysted Artemia embryos. PLoS One. 2013;8(6):e68374. 
37. Iwahori K, Serada S, Fujimoto M, Nomura S, Osaki T, Lee C, et al. Overexpression of SOCS3 exhibits preclinical antitumor activity against malignant pleural mesothelioma. Int J Cancer. 2011;129(4):1005-17.

38. Sarkar S, Hopper AK. tRNA nuclear export in Saccharomyces cerevisiae: In situ hybridization analysis. Mol Biol Cell. 1998;9(11):3041-55.

Submit your next manuscript to BioMed Central and we will help you at every step:

- We accept pre-submission inquiries

- Our selector tool helps you to find the most relevant journal

- We provide round the clock customer support

- Convenient online submission

- Thorough peer review

- Inclusion in PubMed and all major indexing services

- Maximum visibility for your research

Submit your manuscript at www.biomedcentral.com/submit 